\title{
Hematoma extradural de clivus - Relato de casos
}

\author{
Rodrigo Moreira Faleiro ${ }^{1}$, Luanna Rocha Vieira Martins ${ }^{2}$, Geraldo Vítor Cardoso Bicalho ${ }^{3}$
}

Hospital João XXIII, Hospital Felício Rocho, Universidade Federal de Minas Gerais e Fundação Hospitalar do Estado de Minas Gerais, Belo Horizonte, MG, Brasil.

\section{RESUMO}

O clivus é considerado o osso mais forte da base do crânio. Dessa forma, sua lesão sugere trauma de relevante impacto. O hematoma extradural agudo de clivus (HEDAC) é particularmente raro, com poucos casos descritos na literatura. A maioria dos relatos de HEDAC envolve vítimas de colisão com veículos de alta velocidade, sendo comum o acometimento da coluna cervical concomitantemente. Neste artigo, são relatados dois casos de HEDAC. O primeiro envolve um paciente do sexo masculino, 53 anos, com história de queda da própria altura. O segundo também envolve um paciente do sexo masculino, 28 anos, vítima de queda de motocicleta em alta velocidade. Ambos evoluíram com resultados favoráveis.

\section{PALAVRAS-CHAVE}

Fossa craniana posterior, hematoma epidural craniano, traumatismos craniocerebrais.

\section{ABSTRACT}

Clivus extradural hematoma - Cases report

The clivus is considered the strongest bone of the skull base. Thus, his injury suggests trauma of significant impact. The clivus extradural hematoma (HEDAC) is particularly rare, with few cases reported in the literature. Most accounts of victims HEDAC involves collision with high-speed vehicles, which often affects the cervical spine concurrent. In this paper, we report two cases of HEDAC. The first involves a 53 years old male with a history of fall from height. The second also involves a 28 years old male suffered high speed motorcycle accident. Both evolved with favorable results.

\section{KEYWORDS}

Cranial fossa posterior, hematoma epidural cranial, craniocerebral trauma.

1 Chefe do Serviço de Neurocirurgia e Neurologia do Hospital João XXIII e Neurocirurgião do Hospital Felício Rocho, Belo Horizonte, MG, Brasil.

2 Acadêmica de Medicina da Universidade Federal de Minas Gerais (UFMG), Belo Horizonte, MG, Brasil.

3 Neurocirurgião do Hospital Felício Rocho, Belo Horizonte, MG, Brasil. 


\section{Introdução}

O clivus é considerado o osso mais forte da base do crânio. Trata-se de uma região central formada pela fusão do corpo do osso esfenoide e porção basilar do osso occipital. Dessa forma, sua lesão sugere trauma de relevante impacto.

O hematoma extradural agudo de clivus (HEDAC) é particularmente raro, com poucos casos descritos na literatura. ${ }^{1-18}$ A sua ocorrência perfaz um total aproximado de $1,2 \%$ a $12,9 \%$ dos casos de hematoma extradural. ${ }^{2,3}$ Contudo, alguns autores sugerem que esse valor seja subestimado, pois provavelmente há casos não diagnosticados. ${ }^{4}$

A maioria dos casos foi relatada em crianças. Contudo, também há relatos em adultos. ${ }^{3}$ Os pacientes acometidos geralmente são vítimas de acidente com veículos em alta velocidade, sendo o atropelamento de pedestre ou ciclista o meio mais comum. ${ }^{1}$ Há uma forte associação dessa lesão com o acometimento da coluna cervical. A maior parte dos casos relatados foi tratada de forma conservadora, com resultados favoráveis.

A seguir são relatados dois casos clínicos de HEDAC.

\section{Relato dos casos}

\section{Caso 1}

Paciente do sexo masculino, 53 anos, morador de rua, com história de queda da própria altura e convulsões generalizadas após libação etílica. Admitido em Escala de Coma de Glasglow (ECG) 9, isocórico, sem déficits focais.

A tomografia computadorizada (TC) de crânio revelou HEDAC com compressão do quarto ventrículo e hidrocefalia (Figura 1).

Foi implantada derivação ventricular externa, retirada na primeira semana, com pouca necessidade de drenagem liquórica.

Paciente apresentou evolução favorável com resolução do hematoma e da hidrocefalia, Glasgow Outcome Scale 4 três semanas após o trauma e paresia do nervo abducente à esquerda (Figura 2).

\section{Caso 2}

Paciente do sexo masculino, 28 anos, vítima de queda de motocicleta em alta velocidade. Admitido em ECG 4 e isocórico. A investigação radiológica evidenciou fratura transversa do clivus (Figura 3), pequeno HEDAC, hemorragia subaracnóidea traumática e pneumoencéfalo (Figura 4).

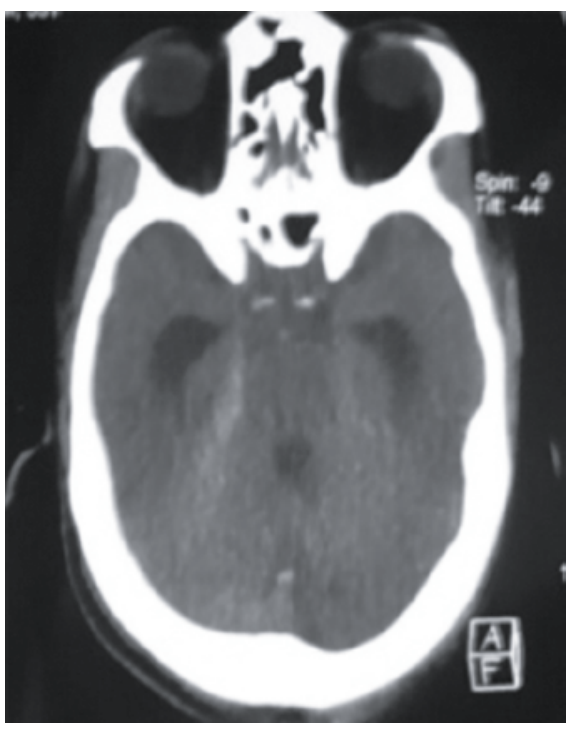

Figura 1 - Tomografia computadorizada (TC) de crânio revela HEDAC, compressão do quarto ventrículo e hidrocefalia.

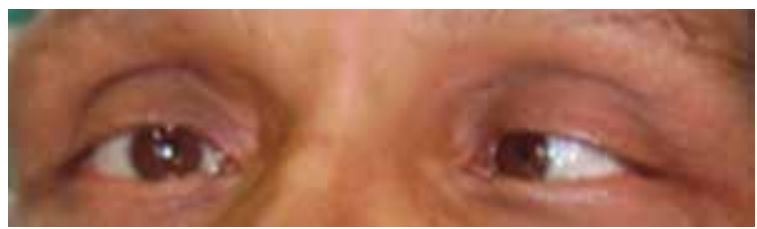

Figura 2 - Paresia do nervo abducente à esquerda.

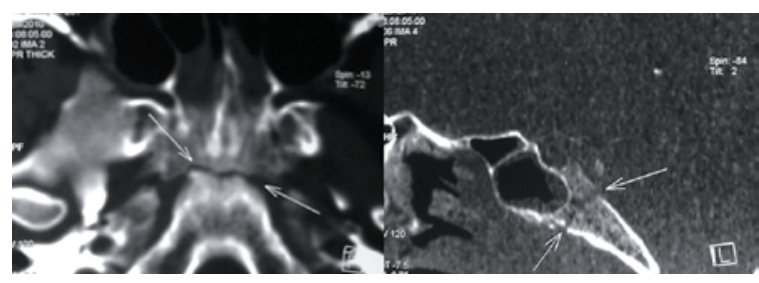

Figura 3 - Tomografia computadorizada (TC) de crânio revela fratura transversa de clivus (setas).

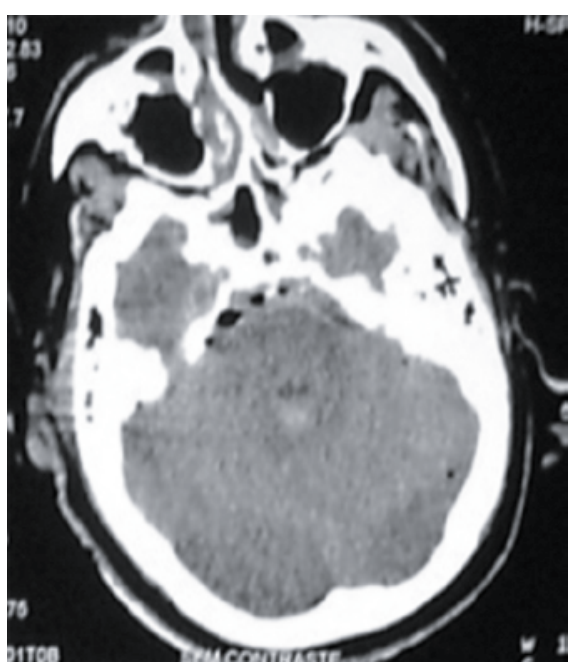

Figura 4 - Tomografia computadorizada (TC) de crânio revela pneumoencéfalo, hemorragia subaracnóidea traumática e pequeno HEDAC. 
Foi submetido à monitoração da pressão intracraniana e a cuidados intensivos.

Apresentou evolução favorável, sem fístula liquórica ou distúrbio endócrino, Glasgow Outcome Scale 4 um mês após trauma, com paresia bilateral do nervo abducente (Figura 5).

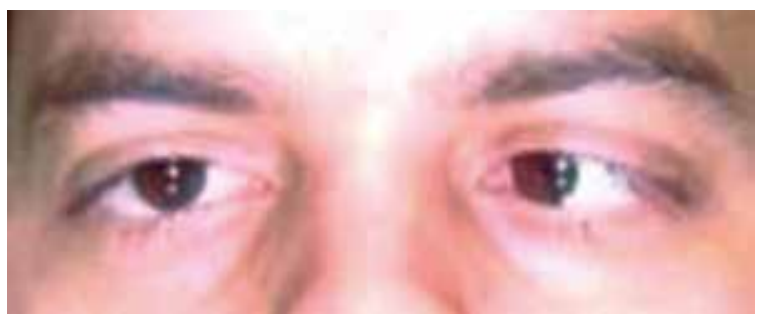

Figura 5 - Paresia bilateral do nervo abducente.

\section{Discussão}

Hematoma extradural é a lesão traumática com efeito de massa mais comum na fossa posterior do crânio. Dentre esse tipo de hematoma, a ocorrência do HEDAC é rara, tendo poucos casos descritos na literatura.

A fisiopatologia do HEDAC ainda não é completamente conhecida. Devido a sua maior ocorrência na faixa etária pediátrica, foi sugerido que características da sua junção craniocervical predispõem à formação dessa lesão. Nesses pacientes, há uma menor estabilidade dessa junção em decorrência do menor tamanho dos côndilos occipitais e de uma horizontalização da articulação atlanto-occipital. ${ }^{1}$ Além disso, a dura-máter de crianças é mais facilmente descolável do crânio, ${ }^{10}$ mecanismo necessário para a formação do hematoma extradural.

O HEDAC separa o clivus da membrana tectorial. Ela mantém uma forte ligação com o clivus e ossos cervicais subjacentes por meio da inserção de tecido conjuntivo rico em estruturas vasculares e neurais. $\mathrm{O}$ deslocamento posterior da membrana tectorial pode ocasionar ruptura desses vasos, acarretando, dessa forma, acúmulo de sangue na área retroclival com consequente formação do hematoma. ${ }^{19}$ Além disso, deslocamento atlanto-occipital ou atlanto-axial em decorrência da hiperextensão ou hiperflexão craniocervical também é reportado, ${ }^{3,16,17} \mathrm{em}$ associação ao HEDAC, assim como luxação anterior ou posterior do processo odontoide com ruptura do ligamento transverso do atlas. ${ }^{10}$

A paralisia do nervo abducente tem sido frequentemente relatada em associação ao HEDAC, assim como foi visto nos casos deste artigo. Há uma relação entre o trajeto do VI par craniano com o clivus e com a membrana tectorial. Dessa forma, a orientação anatômica do nervo abducente e sua interação com o ápice petroso o predispõem à lesão.

Os pacientes vítimas de HEDAC geralmente apresentam múltiplos traumas associados e estão graves clinicamente, uma vez que a formação dessa lesão envolve mecanismos de grande impacto. Em decorrência de sua localização na fossa posterior do crânio, esse hematoma pode ocasionar compressão direta do tronco encefálico com as consequentes repercussões clínicas. No primeiro caso relatado, no entanto, o hematoma decorreu de trauma de baixo impacto e não foi observada fratura de clivus. Provável discrasia sanguínea pelo etilismo pode ter participado na formação do hematoma.

Para o diagnóstico de HEDAC, é necessário um alto índice de suspeição clínica. Além disso, em virtude de artefatos ósseos na base do crânio, a tomografia computadorizada (TC) convencional pode não detectar o hematoma. Por essa razão, a TC multi-slice e a reconstrução sagital devem ser consideradas quando se suspeita de HEDAC (Figura 6). A ressonância magnética é superior à TC no diagnóstico do hematoma retroclival. Entretanto, nem sempre está disponível ou indicada no paciente politraumatizado. ${ }^{15}$

A maioria dos casos de HEDAC relatados na literatura foi tratada conservadoramente, com resultados favoráveis. Tratamento cirúrgico foi relatado em alguns casos, sendo descrita a abordagem por via transoral ${ }^{9} \mathrm{e}$ por descompressão posterior, ${ }^{12}$ tendo bons resultados.

A literatura, ${ }^{20}$ assim como nossos casos, evidencia que não se pode estabelecer uma correlação entre a ECG do paciente no momento de sua admissão e os resultados finais. Os casos 1 e 2 relatados neste artigo, por exemplo, possuíam ECG 9 e 4, respectivamente, e evoluíram com resultados favoráveis.

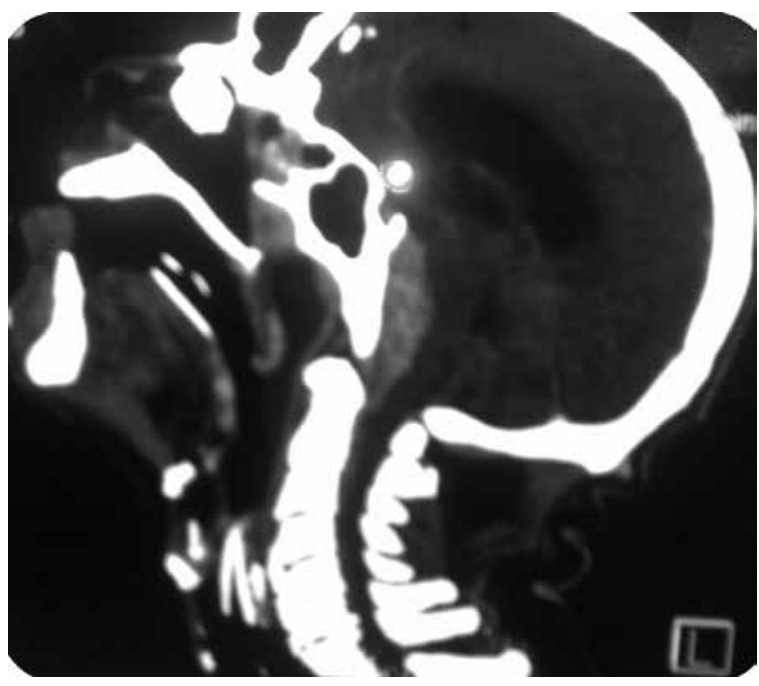

Figura 6 - Tomografia computadorizada (TC) com reconstrução sagital revela HEDAC. 
Além disso, como comprovado pelos nossos casos, apesar de sua maior ocorrência na faixa etária pediátrica, o HEDAC não é exclusivo de crianças e deve ser considerado sempre que houver paralisia bilateral de nervos cranianos, em especial do VI par, em um contexto de traumatismo craniano. $^{18}$

\section{Conflito de interesses}

Os autores declaram não haver conflito de interesses neste trabalho.

\section{Referências}

1. Tubbs RS, Griessenauer CJ, Hankinson T, Rozzelle C, Wellons JC 3rd, Blount JP, et al. Retroclival epidural hematomas: a clinical series. Neurosurgery. 2010;67(2):404-6.

2. Agrawal D, Cochrane DD. Traumatic retroclival epidural hematoma - A pediatric entity? Childs Nerv Syst. 2006;22(7):670-3.

3. Ratilal B, Castanho P, Vara Luiz C, Antunes JO. Traumatic clivus epidural hematoma: case report and review of the literature. Surg Neurol. 2006;66(2):200-2.

4. Yama N, Kano H, Nara S, Kurimoto Y, Narimatsu E, Koito $\mathrm{K}$, et al. The value of multidetector row computed tomography in the diagnosis of traumatic clivus epidural hematoma in children: a three-year experience. J Trauma. 2007;62(4):898-901.

5. Guillaume D, Menezes AH. Retroclival hematoma in the pediatric population. Report of two cases and review of the literature. J Neurosurg. 2006;105(Suppl 4):321-5.

6. Castillo C, Lambré H, Meli F, Boero A, Lylyk P. Acute extraaxial hematoma post-traumatic of the clivus. Medicina (B Aires). 1994;54(2):150-2.

7. Kurosu A, Amano K, Kubo O, Himuro H, Nagao T, Kobayashi $\mathrm{N}$, et al. Clivus epidural hematoma. Case Report. J Neurosurg. 1990;72(4):660-2.
8. Kwon TH, Joy H, Park YK, Chung HS. Traumatic retroclival epidural hematoma in a child: case report. Neurol Med Chir (Tokyo). 2008;48(8):347-50.

9. Marks SM, Paramaraswaren RN, Johnston RA. Transoral evacuation of a clivus extradural haematoma with good recovery: a case report. Br J Neurosurg. 1997;11(3):245-7.

10. Mizushima H, Kobayashi N, Sawabe Y, Hanakawa K, Jinbo $\mathrm{H}$, lida $\mathrm{M}$, et al. Epidural hematoma of the clivus. Case report. J Neurosurg. 1998;88(3):590-3.

11. Müller JU, Piek J, Kallwellis G, Stenger RD. Prepontine epidural hemorrhage. Zentralbl Neurochir. 1998;59(3):185-8.

12. Orrison WW, Rogde S, Kinard RE, Williams JE, Torvik A, Sackett JF, et al. Clivus epidural hematoma: a case report. Neurosurgery. 1986;18(2):194-6.

13. Papadopoulos SM, Dickman CA, Sonntag VK, Rekate HL, Spetzler RF. Traumatic atlantooccipital dislocation with survival. Neurosurgery. 1991;28(4):574-9.

14. Paterakis $\mathrm{KN}$, Karantanas AH, Hadjigeorgiou GM, Anagnostopoulos V, Karavelis A. Retroclival epidural hematoma secondary to a longitudinal clivus fracture. Clin Neurol Neurosurg. 2005;108(1):67-72

15. Suliman HM, Merx HL, Wesseling P, van der Sluijs B, Vos $\mathrm{PE}$, Thijssen HO. Retroclival extradural hematoma is a magnetic resonance imaging diagnosis. J Neurotrauma. 2001;18(11):1289-93.

16. Vera M, Navarro R, Esteban E, Costa JM. Association of atlanto-occipital dislocation and retroclival haematoma in a child. Childs Nerv Syst. 2007;23(8):913-6.

17. Yang BP. Traumatic retroclival epidural hematoma in a child. Pediatr Neurosurg. 2003;39(6):339-40.

18. Zuccarello M, Pardatscher K, Andrioli GC, Fiore DL, lavicoli $\mathrm{R}$, et al. Epidural hematomas of the posterior cranial fossa. Neurosurgery. 1981;8(4):434-7.

19. Tubbs RS, Kelly DR, Humphrey ER, Chua GD, Shoja MM, Salter EG, et al. The tectorial membrane: anatomical, biomechanical, and histological analysis. Clin Anat. 2007;20(4):382-6.

20. laconetta G, Fusco M, Cavallo LM, Cappabianca P, Samii M, Tschabitscher M. The abducens nerve: microanatomic and endoscopic study. Neurosurgery. 2007;61(Suppl 3):7-14.

Endereço para correspondência

Rodrigo Moreira Faleiro

Rua Caraça, 518, ap. 201, Serra

30220-260 - Belo Horizonte, MG, Brasil

Telefone: (31) 9970-7676

E-mail: r.m.faleiro@hotmail.com 